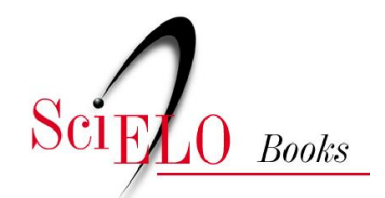

\title{
Serviços de governo eletrônico um panorama do uso no Brasil
}

\author{
Ernani Marques dos Santos \\ Nicolau Reinhard
}

\section{SciELO Books / SciELO Livros / SciELO Libros}

SANTOS, E.M., and REINHARD, N. Serviços de governo eletrônico: um panorama do uso no Brasil. In: PINHO, J.A.G., ed. Artefatos digitais para mobilização da sociedade civil: perspectivas para avanço da democracia [online]. Salvador: EDUFBA, 2016, pp. 223-245. ISBN: 978-85-232-1877-5. https://doi.org/10.7476/9788523218775.0010.

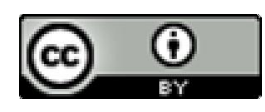

All the contents of this work, except where otherwise noted, is licensed under a Creative Commons Attribution $\underline{4.0 \text { International license. }}$

Todo o conteúdo deste trabalho, exceto quando houver ressalva, é publicado sob a licença Creative Commons Atribição 4.0.

Todo el contenido de esta obra, excepto donde se indique lo contrario, está bajo licencia de la licencia Creative Commons Reconocimento 4.0 . 


\section{SERVIÇOS DE GOVERNO ELETRÔNICO}

um panorama do uso no Brasil

ERNANI MARQUES DOS SANTOS, NICOLAU REINHARD

INTRODUÇÃO

Um dos principais objetivos da implementação do governo eletrônico é proporcionar o acesso às informações e serviços públicos demandados pela sociedade em geral, de forma a satisfazer as necessidades dos usuários e suas expectativas.

A forma de operacionalização do governo eletrônico é por meio da prestação de serviços eletrônicos - serviços que são oferecidos, fornecidos e/ou consumidos pela internet. (SWID; ELMELEGY, 2012)

No entanto, embora tenha sido cada vez mais crescente o acesso às tecnologias de informação e comunicação, notadamente em relação à internet, entre a oferta e o uso desses serviços nem sempre existe uma correlação direta. A adoção e o uso dos serviços dependem de muitos fatores, dentre eles a utilidade percebida, percepção de facilidade de uso, preocupações de risco inerente (HOFFMAN; NOVAK; PERALTA, 1999) e confiança. (LEE; KIM; AHN, 2011) Além disso, a implementação de governo eletrônico envolve muitos grupos de diferentes stakeholders, que precisam ser levados em consideração no desenvolvimento dos serviços a serem disponibilizados. (FLAK et al., 
2007; TRANMÜLLER; WIMMER, 2000) Janssen e Cresswell (2005), e Kamal, Weerakkody e Irani (2011) também enfatizam que as iniciativas de governo eletrônico envolvem iniciativas de stakeholders de dentro e de fora da organização.

A partir desse cenário, o objetivo do presente artigo é analisar o uso do governo eletrônico no Brasil, a fim de traçar um perfil da utilização desses serviços e suas motivações para o não uso. Para tanto, utiliza-se os dados coletados nas pesquisas TIC Domicílios 2009 a 2013, realizadas pelo Centro Regional de Estudos para o Desenvolvimento da Sociedade da Informação (CETIC.br), do Núcleo de informação e Coordenação do Ponto BR (NIC.br) - braço executivo do Comitê Gestor da Internet no Brasil (CGI.br). Esta pesquisa investiga a posse e o uso das Tecnologias de Informação e Comunicação (TICs) no Brasil, em várias áreas, sendo que aqui foca-se especificamente nos dados referentes a governo eletrônico. Embora seja senso comum que esses serviços podem trazer benefícios para a sociedade e também para o governo, seu uso ainda pode ser considerado pequeno frente ao potencial e importância.

\section{GOVERNO ELETRÔNICO}

Para Grande, Araujo e Serna (2002) o termo governo eletrônico não possui uma clara definição. Várias conceituações, notadamente de instituições, são encontradas: National Audit Office (2002), Organização para a Cooperação e Desenvolvimento Econômico (2001), United Nations (2001), European Public Administration Network (2004), Intosai (2003) e Okot-Uma (2001), entre outras.

Para o World Bank (2003),

governo eletrônico refere-se ao uso, por agências governamentais, de tecnologias de informação (como redes de longa distância, internet e computação móvel) capazes de transformar as relações com cidadãos, empresas e outras unidades do governo. Essas tecnologias podem servir a diferentes fins, como: melhor prestação de serviços aos cidadãos, interações mais eficazes com empresas e a indústria, empowerment do cidadão por meio do acesso a informações ou mais eficiência na administração governamental. 
Criado e Ramilo (2001) apresentam uma definição mais ampla, referindo-se à adoção de Tecnologias de Informação e Comunicação pela Administração Pública como diferentes vias através das quais organizações se conectam e interagem com outras organizações e pessoas, especialmente mediante suas páginas web, mas também através de correio eletrônico e outras ferramentas como telefone e outros dispositivos móveis, vídeo conferência, intranets privadas, extranets, TV a cabo, ondas de rádio e satélites.

Em linhas gerais, as funções características do governo eletrônico são (SANTOS; REINHARD, 2012, p. 121-122):

- prestação eletrônica de informações e serviços;

- regulamentação das redes de informação, envolvendo principalmente governança, certificação e tributação;

- prestação de contas públicas, transparência e monitoramento da execução orçamentária;

- ensino a distância, alfabetização digital e manutenção de bibliotecas virtuais;

- difusão cultural com ênfase nas identidades locais, fomento e preservação das culturas locais;

- e-procurement, isto é, aquisição de bens e serviços por meio da internet, como licitações públicas eletrônicas, pregões eletrônicos, cartões de compras governamentais, bolsas de compras públicas virtuais e outros tipos de mercados digitais para bens adquiridos pelo governo;

- estímulo aos negócios eletrônicos, através da criação de ambientes de transações seguras, especialmente para pequenas e médias empresas.

Essas funções, ao serem realizadas, envolvem basicamente quatro tipos de transações (SANTOS; REINHARD, 2012, p. 122):

$\mathrm{G}_{2} \mathrm{G}$, quando se trata de uma relação intra ou inter-governos; $\mathrm{G} 2 \mathrm{~B}$, caracterizado por transações entre governos e pessoas jurídicas em seus vários papéis desempenhados; e $\mathrm{G} 2 \mathrm{C}$, envolvendo relações entre governos e cidadãos; e G2E, relacionadas às transações entre governos e seus próprios servidores. 
A implementação de governo eletrônico é um processo que pode apresentar estágios distintos, tendo em vista os níveis de informações e serviços prestados. Várias classificações têm sido formuladas, como, por exempo, Gartner Group - Baum e Maio (2000), Deloitte e Touche (2001), Hiller (2001), Layne e Lee (2001), Moon (2002), United Nations (2001) e Siau e Long (2004).

O modelo proposto pelas Nações Unidas (2001), estabelece cinco estágios: (1) presença emergente; (2) presença aprimorada; (3) presença interativa; (4) presença transacional e; (5) presença em rede. Esse último estágio é definido como uma estrutura $\mathrm{G}_{2} \mathrm{C}$ (governo para cidadão) baseada em uma rede integrada de agências públicas para a provisão de informação, conhecimento e serviços (Quadro 1).

Quadro 1 - Estágios de governo eletrônico

\begin{tabular}{|c|c|}
\hline ESTÁGIO & CARACTERÍSTICAS \\
\hline $\begin{array}{l}\text { I - Presença } \\
\text { Emergente }\end{array}$ & $\begin{array}{l}\text { Presença na web por meio de um website oficial, um portal nacional ou } \\
\text { uma home page oficial; links para os ministérios do governo, governos } \\
\text { estaduais e locais, administração indireta; a informação é limitada, } \\
\text { básica e estática. }\end{array}$ \\
\hline $\begin{array}{l}\text { II - Presença } \\
\text { Aprimorada }\end{array}$ & $\begin{array}{l}\text { Serviços on-line são aprimorados para incluir bases de dados e fontes } \\
\text { de informações atuais e arquivadas, como políticas, leis e regulação, } \\
\text { relatórios, informativos e bases de dados para serem baixadas pela } \\
\text { internet. O usuário pode procurar por um documento e há função de } \\
\text { ajuda e um mapa do sítio é disponibilizado. }\end{array}$ \\
\hline $\begin{array}{l}\text { III - Presença } \\
\text { Interativa }\end{array}$ & $\begin{array}{l}\text { Provisão de serviços on-line do governo entra no modo interativo; } \\
\text { facilidades para download online; link seguro; facilidade de assinatura } \\
\text { eletrônica; capacidade de áudio e vídeo para informação pública } \\
\text { relevante. Os servidores públicos podem ser contactados via e-mail, } \\
\text { fax, telefone e correio. O sítio é atualizado com grande regularidade. }\end{array}$ \\
\hline $\begin{array}{l}\text { IV - Presença } \\
\text { Transacional }\end{array}$ & $\begin{array}{l}\text { Usuários têm condições de conduzir transações on-line, como pagar } \\
\text { multas por transgressões no trânsito, impostos e taxas por serviços } \\
\text { postais por meio de cartão de crédito, bancário ou de débito. Há } \\
\text { algumas facilidades para licitar on-line em contratações públicas via } \\
\text { links seguros. }\end{array}$ \\
\hline $\begin{array}{l}\text { V - Presença } \\
\text { em Rede }\end{array}$ & $\begin{array}{l}\text { Uma estrutura } \mathrm{G}_{2} \mathrm{C} \text { (governo para cidadão) baseada em uma rede } \\
\text { integrada de agências públicas para a provisão de informação, } \\
\text { conhecimento e serviços. A ênfase está no feedback para o governo. } \\
\text { Um formulário de comentário na web é disponibilizado. Um calendário } \\
\text { de eventos governamentais futuros existe com um convite do governo } \\
\text { à participação. Governo solicita feedback por meio de mecanismos } \\
\text { de pesquisa de opinião on-line; fóruns de discussão; e facilidades de } \\
\text { consulta on-line. }\end{array}$ \\
\hline
\end{tabular}

Fonte: United Nations (2001). 
O estágio de presença em rede, como mencionado acima, pressupõe integração de sistemas antigos e desenvolvimento e implementação de novos sistemas dentro dessa perspectiva. Mas segundo Avgerou e colaboradores (2005), o suporte aos serviços de governo requer a transformação de sistemas legados implantados nas administrações públicas burocráticas em sistemas de informações modernos, o que se constitui numa tarefa bastante difícil, tanto no aspecto tecnológico como organizacional. Tecnologicamente, o desafio compreende a modernização e integração de sistemas fragmentados para formar uma infraestrutura capaz de suportar melhorias na prestação de serviços, como, por exemplo, possibilitar a redução ou eliminação da necessidade de acessar múltiplos órgãos do governo a fim de obter um serviço. (CIBORRA; NAVARRA, 2003) No plano organizacional, Avgerou e colaboradores (2005) argumentam que não existe um método instantâneo para se conseguir as mudanças necessárias para criar a capacidade da administração em melhorar a disponibilização de serviços públicos a longo prazo.

Segundo Oliveira (2003), no último estágio de implantação do governo eletrônico, as aplicações tornam-se mais avançadas e, ao efetuar uma transação com o governo, o usuário não precisa saber quais são os órgãos ou departamentos, de quais níveis de governo e em que sequência são mobilizados para obtenção de determinado serviço ou informação. O processamento das transações é feito de forma total através de um ambiente, provendo ao usuário a informação ou serviço solicitado por meio de um único ponto de acesso, o que tem sido denominado como "governo de parada única" ou "de janela única" (dos termos em inglês on-stop government e single-window service, respectivamente). (BENT; KERNAGHAN; MARSON, 1999; HAGEN; KUBICEK, 2000)

Corforme Santos e Reinhard (2012), de uma forma simplificada, a implementação de governo eletrônico pode se apresentar em três formas: presença na internet através de informações básicas, capacidades de transação para indivíduos e empresas e informações e transações integradas, com a colaboração entre diversas agências (janela única / governo de parada única). Os autores frisam, no entanto, que a con- 
cretização do último estágio envolve desafios políticos, estratégicos e procedimentais, nos quais a cooperação interagências é fundamental, além dos aspectos tecnológicos.

\section{METODOLOGIA}

O presente artigo baseia-se em dados coletados pelo Centro Regional de Estudos para o Desenvolvimento da Sociedade da Informação (CETIC.br), do Núcleo de informação e Coordenação do Ponto BR (NIC. br) - braço executivo do Comitê Gestor da Internet no Brasil (CGI.br), em sua pesquisa anual TIC Domicílios, ${ }^{\mathrm{I}}$ no período 2009-2013.

Os dados foram coletados de acordo com os padrões metodológicos e indicadores estabelecidos pela International Telecommunication Union (ITU), Organização das Nações Unidas (ONU), utilizando como referência o Manual for Measuring ICT Acess and Use by Households and Individuals. (ITU, 2014) Também foram observadas as orientações da OECD e do Instituto de Estatísticas da Comissão Europeia (Eurostat). Além disso, foram levadas em conta as orientações contidas nos documentos metodológicos produzidos pelo Observatório para a Sociedade da Informação na América Latina e Caribe (OSILAC), da Comissão Econômica para a América Latina e Caribe das Nações Unidas (CEPAL). O plano amostral utilizou informações do Censo Demográfico Brasileiro 2000 e 2010 (IBGE, 2000, 2010) e da Pesquisa Nacional por Amostra de Domicilio - PNAD 2008 a 2012 (IBGE, 2008, 2009, 2010, 2011, 2012), ambas realizadas pelo Instituto Brasileiro de Geografia e Estatística (IBGE). Na pesquisa TIC Domicílios 2013, as fontes para o desenho amostral foram as seguintes (Figura 1).

A pesquisa mede a disponibilidade e o uso das TICs e da internet em diversas áreas, sendo o questionário estruturado em blocos de perguntas, cobrindo os tópicos de acesso às TICs, uso do computador, uso da internet, segurança na rede, uso do e-mail, spam, governo eletrônico, comércio eletrônico, habilidades com o computador/internet, aces-

I A pesquisa TIC Domicílios é realizada anualmente desde 2005 e publicada no mês de junho do ano seguinte; no momento da elaboração deste artigo a última pesquisa disponível era a de 2013 , publicada em junho de 2014 . 
so sem fio (uso do celular) e intenção de aquisição de equipamentos e serviços TIC, além de um bloco inicial para caracterização socioeconômica dos respondentes. Para este artigo, foram utilizados apenas os dados referentes ao uso de serviços de governo eletrônico relativos às transações entre Governo e Cidadãos $\left(\mathrm{G}_{2} \mathrm{C}\right)$, considerando-se os usuários com 16 anos de idade ou mais, além de alguns dados do perfil socioeconômico dos pesquisados.

Figura 1 - Desenho amostral da pesquisa

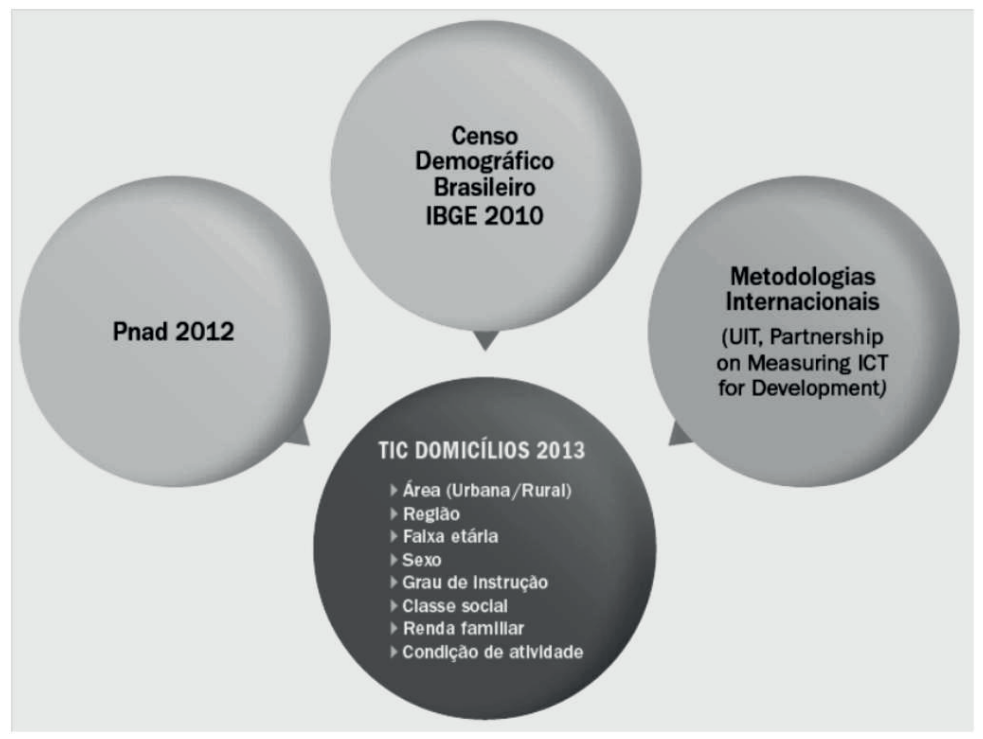

Fonte: CGI.br (2014).

\section{APRESENTAÇÃO E DISCUSSÃO DOS DADOS}

A título de caracterização dos seus respondentes, a pesquisa TIC domicílios 2013 apresentou os seguintes dados (CGi, 2014):

- distribuição geográfica: $43 \%$ pertenciam à região Sudeste; $27 \%$ à Nordeste; $15 \%$ à Sul; 8\% à Norte; e 7\% à Centro-Oeste.

- sexo: $52 \%$ eram do sexo feminino e $48 \%$ do sexo masculino. 
- grau de isntrução: $49 \%$ estavam cursando o Ensino Fundamental; 31\% o Ensino Médio; 13\% possuem o Ensino Superior; e 7\% eram analfabetos ou cursaram apenas a Educação Infantil.

- idade: $12 \%$ possuíam entre 10 e 15 anos; $17 \%$ entre 16 e 24 anos; $19 \%$ entre 25 e 34 anos; $17 \%$ entre 35 e 44 anos; $20 \%$ entre 45 e 59 anos; e $15 \% 60$ anos ou mais.

- distribuição de renda: $14 \%$ declararam possuir renda familiar de até um salário mínimo; 30\%, mais de um até dois salários mínimos; $21 \%$ declararam ter mais de dois até três salários mínimos; 13\%, mais de três a cinco salários mínimos; 7\%, mais de cinco até dez salários mínimos; e 4\%, mais de dez salários mínimos; $11 \%$ da amostra não souberam ou se recusaram a responder a renda familiar total do domicílio onde residem.

- classe econômica (segundo o Critério Brasil da Abep): 49\% da amostra pertencia à classe $\mathrm{C} ; 27 \%$ à classe $\mathrm{B} ; 22 \%$ às classes $\mathrm{D}$ e E; e apenas $2 \%$ à classe $\mathrm{A}$.

O uso de internet por parte dos pesquisados é apresentado no Gráfico 1, em relação à área de uso, região demográfica, sexo, nível de instrução, idade e classe econômica. Dois indicadores que chamam a atenção: o de instrução, com 93\% no nível superior e o de classe social, com $97 \%$ na classe A.

Segundo o CGi (2014, p. 176):

A massiva presença de computador e conexão de Internet em domicílios da classe A contribui para o amplo uso da rede entre os indivíduos dessa classe: $97 \%$ deles estavam conectados em 2013. Na classe B, a proporção de usuários também é alta, chegando a $78 \%$. Entretanto, permanece o desafio de universalização nas classes C (49\%) e, sobretudo, D e E (17\%). 
Gráfico 1 - Proporção de indivíduos que utilizaram internet - percentual sobre o total da população

\begin{tabular}{|c|c|c|}
\hline \multicolumn{2}{|l|}{ TOTAL } & 51 \\
\hline \multirow{2}{*}{ Área } & Urbana & 56 \\
\hline & Rural & 21 \\
\hline \multirow{5}{*}{ Região } & Sudeste & 56 \\
\hline & Nordeste & 42 \\
\hline & Sul & 56 \\
\hline & Norte & 39 \\
\hline & Centro-Oeste & 58 \\
\hline \multirow{2}{*}{ Sexo } & Masculino & 49 \\
\hline & Feminino & 53 \\
\hline \multirow{4}{*}{ Grau de instrução } & Analfabeto / Educação infantil & 2 \\
\hline & Fundamental & 32 \\
\hline & Médio & 74 \\
\hline & Superior & 93 \\
\hline \multirow{6}{*}{ Faixa etária } & De 10 a 15 anos & 75 \\
\hline & De 16 a 24 anos & 77 \\
\hline & De 25 a 34 anos & 66 \\
\hline & De 35 a 44 anos & 47 \\
\hline & De 45 a 59 anos & 33 \\
\hline & 60 anos ou mais & 11 \\
\hline \multirow{4}{*}{ Classe social } & DE & 17 \\
\hline & C & 49 \\
\hline & B & 78 \\
\hline & A & 97 \\
\hline
\end{tabular}

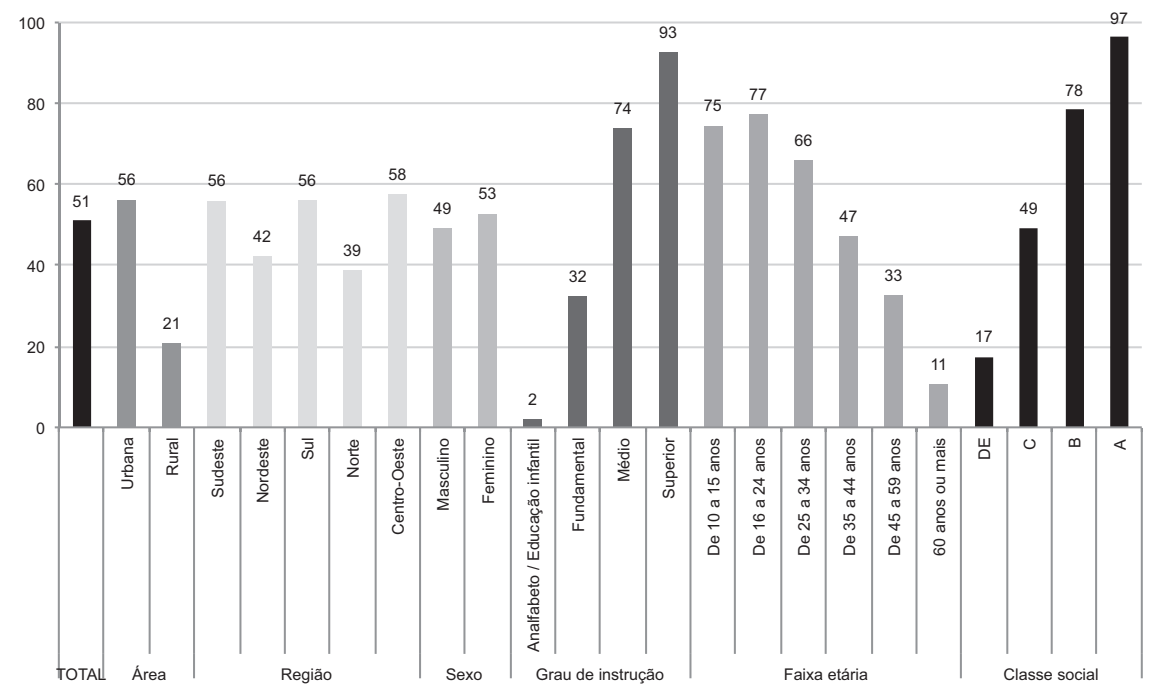

Fonte: CGI.br (2014). 
Ainda par o CGi (2014, p. 176):

Em números absolutos, o Brasil tem 69,9 milhões de pessoas a partir de 10 anos de idade que nunca usaram a Internet, sendo que a concentração maior está entre os brasileiros com mais de 45 anos - o equivalente a 42,6 milhões de pessoas. A mesma desigualdade pode ser observada por renda. Indivíduos que residem em domicílios com renda média familiar que não ultrapassa dois salários mínimos representam 43 milhões de brasileiros excluídos digitalmente. Entre os analfabetos, $97 \%$ nunca utilizaram a Internet, proporção que atinge $60 \%$ entre os que estudaram até o Ensino Fundamental.

O uso da internet pelos respondentes apresentou uma distribuição entre diversas atividades, conforme o Gráfico 2, sendo a participação em redes sociais a que mais se destacou.

Gráfico 2 - Proporção de usuários de internet por atividade realizada percentual sobre o total de usuários de internet

\begin{tabular}{|l|l|}
\hline Participar de redes sociais, como Facebook, Orkut ou Google + & 77 \\
\hline Enviar mensagens instantâneas, por exemplo, por chat do Facebook, chat do Skype & 74 \\
\hline Enviar e receber e-mail & 72 \\
\hline Procurar informações sobre produtos e serviços & 65 \\
\hline Ouvir música on-line & 63 \\
\hline Compartilhar conteúdo na Internet, como textos, imagens ou vídeos & 60 \\
\hline Assistir filmes ou vídeos (em sites como o YouTube) & 56 \\
\hline Realizar atividades ou pesquisas escolares & 55 \\
\hline Baixar ou fazer o download de músicas & 50 \\
\hline
\end{tabular}




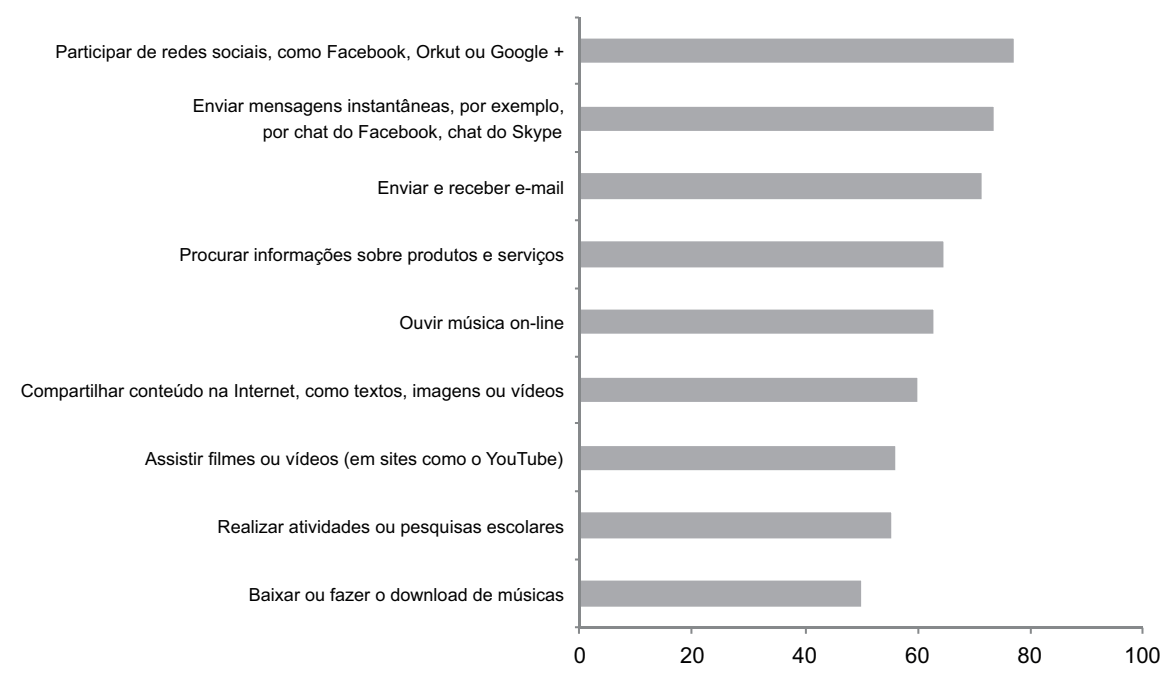

Fonte: CGI.br (2014).

Para analisar as atividades relacionadas ao governo eletrônico, a pesquisa TIC Domicílios considera os indivíduos com 16 anos de idade ou mais que utilizaram a internet nos três meses anteriores à entrevista, avaliando uma série de 22 tipos de serviços selecionados, relacionados a ações de busca de informação e transações realizadas. Os dados relativos a estes serviços estão na Tabela 1.

Em 2013, 68\% dos indivíduos declararam ter utilizado alguns dos serviços de governo eletrônico pesquisados. Segundo os dados coletados, os usuários que utilizam tais serviços com mais frequência possuem o Ensino Superior (86\%), têm idade entre 35 e 44 anos (74\%) e são pertencentes à classe A (87\%). Segundo o CGi (2014), a estivativa dos usuários de algum dos serviços de governo eletrônico pesquisados pela TIC Domicílios é de cerca de 48,4 milhões. 
Tabela 1 - Serviços de governo eletrônico utilizados

\begin{tabular}{|c|c|c|c|}
\hline \multirow{2}{*}{ SERVIÇOS UTILIZADOS } & \multicolumn{3}{|c|}{ \% POR ÁREA } \\
\hline & URBANA & RURAL & TOTAL \\
\hline \multicolumn{4}{|l|}{ 1. Obtenção de documentos } \\
\hline Consulta de CPF & 28 & 12 & 27 \\
\hline $\begin{array}{l}\text { Busca de informações sobre emissão de documentos } \\
\text { (RG, CPF, etc.) }\end{array}$ & 28 & 16 & 27 \\
\hline $\begin{array}{l}\text { Emissão de documentos (certificados, atestados, } \\
\text { comprovantes, etc.) }\end{array}$ & 18 & 14 & 18 \\
\hline $\begin{array}{l}\text { Obtenção de certidões negativas, licenças e } \\
\text { permissões }\end{array}$ & 16 & 06 & 15 \\
\hline \multicolumn{4}{|l|}{ 2. Pagamentos de taxas e impostos } \\
\hline Declaração de imposto de renda (inclusive isentos) & 20 & 12 & 20 \\
\hline Obtenção informação sobre impostos e taxas & 24 & 15 & 23 \\
\hline Pagamento de impostos, multas e taxas & 21 & 14 & 21 \\
\hline \multicolumn{4}{|l|}{ 3. Previdência e Assistência Social } \\
\hline $\begin{array}{l}\text { Busca de informações sobre Previdência Social e } \\
\text { benefícios sociais }\end{array}$ & 18 & 12 & 17 \\
\hline Solicitação de serviços junto à Previdência Social & ०9 & ०4 & ०9 \\
\hline \multicolumn{4}{|l|}{ 4. Justiça e Segurança } \\
\hline Consulta de pontos na carteira de habilitação /multas & 17 & 13 & 17 \\
\hline Busca de informações sobre direito do consumidor & 20 & 12 & 19 \\
\hline $\begin{array}{l}\text { Consulta de andamento de atos processuais na } \\
\text { justiça }\end{array}$ & 12 & 05 & 12 \\
\hline Busca de informações sobre veículos roubados & 05 & 07 & 05 \\
\hline Boletim de ocorrência & 06 & 02 & 06 \\
\hline \multicolumn{4}{|l|}{ 5. Outros Serviços } \\
\hline $\begin{array}{l}\text { Busca de informações sobre serviços públicos de } \\
\text { educação }\end{array}$ & 25 & 23 & 25 \\
\hline Inscrição em concursos públicos & 24 & 14 & 23 \\
\hline Busca de informações sobre empregos & 18 & ०9 & 17 \\
\hline Busca de informações sobre direitos do trabalhador & 25 & 16 & 24 \\
\hline $\begin{array}{l}\text { Busca de informações sobre serviços públicos de } \\
\text { saúde }\end{array}$ & 21 & 14 & 21 \\
\hline
\end{tabular}




\begin{tabular}{|c|c|c|c|}
\hline Matrículas em escolas ou instituições de ensino & 10 & 08 & 10 \\
\hline Marcação e/ou agendamento de consultas médicas & 10 & $\mathrm{O} 2$ & 10 \\
\hline $\begin{array}{l}\text { Participação em fóruns, chats, votações etc. } \\
\text { relacionados ao governo }\end{array}$ & 08 & 05 & 08 \\
\hline
\end{tabular}

Fonte: Elaborado pelos autores a partir de dados de CGI.br (2014).

Conforme dados constantes na Tabela 1, dos 22 serviços relacionados nenhum deles apresenta índice de utilização por pelo menos metade dos respondentes. Entre as atividades de governo eletrônico investigadas pela pesquisa TIC Domicílios 2013, em nível Brasil, considerando-se as áreas urbana e rural, a consulta ao Cadastro de Pessoa Física (CPF) foi realizada por $27 \%$ dos usuários. A busca de informações sobre emissão de documentos (RG, CPF, etc.) também obteve $27 \%$, e a busca de informações sobre serviços públicos de educação foi mencionada por $25 \%$. Já o pagamento de impostos, multas ou taxas foram feitos por $21 \%$ deles. Por outro lado, algumas atividades tiveram baixo índice de uso, tais como busca de informações sobre veículos roubados (5\%), fazer boletim de ocorrência (6\%) e participar de fóruns, chats e votações relacionados ao governo (8\%). Um ponto a se destacar é que os serviços que apresentaram maior índice de utilização são característicos ainda dos estágios 1 (presença emergente) e 2 (presença aprimorada) da classificação de níveis de maturidade proposta pelas Nações Unidas. (UNITED NATIONS, 200I) São serviços basicamente de obtenção de informações, com baixo nível de transações com interações efetivas entre usuários e Governo.

Segundo as pesquisas, o uso de serviços de governo eletrônico vem crescendo no período 2009-2013. Considerando aqueles que já utilizaram a internet pelo menos uma vez, 68\% usaram algum serviço durante 2013 , o que representa um aumento de $3 \%$ em relação a 2012 , e cerca de $152 \%$ em relação a 2009 (Tabela 2). Essa evolução, inclusive no que se refere aos dados específicos das áreas estudadas, pode ser visualizada no Gráfico 3. 
Tabela 2 - Proporção de indivíduos que usaram governo eletrônico no período 2009-2013

\begin{tabular}{|c|c|c|c|}
\hline \multicolumn{4}{|c|}{ \% EM RELAÇÃO AOS USUÁRIOS DE INTERNET } \\
\hline \multirow{2}{*}{ ANO } & \multicolumn{3}{|l|}{ ÁREA } \\
\hline & TOTAL & URBANA & RURAL \\
\hline 2009 & 27 & 30 & 10 \\
\hline 2010 & 23 & 26 & 07 \\
\hline 2011 & 31 & 34 & 10 \\
\hline 2012 & 65 & 66 & 50 \\
\hline 2013 & 68 & 69 & 52 \\
\hline
\end{tabular}

Fonte: elaborado pelos autores a partir de dados de CGI.br (2010, 2011, 2012, 2013, 2014).

Gráfico 3 - Uso de governo eletrônico no período 2009-2013 percentual em relação ao número de usuários de internet

\begin{tabular}{|l|l|l|l|}
\hline \multicolumn{4}{|l|}{ \% em relação aos usuários de internet } \\
\hline Ano & Total & Urbana & Rural \\
\hline 2009 & 27 & 30 & 10 \\
\hline 2010 & 23 & 26 & 7 \\
\hline 2011 & 31 & 34 & 10 \\
\hline 2012 & 65 & 66 & 50 \\
\hline 2013 & 68 & 69 & 52 \\
\hline
\end{tabular}

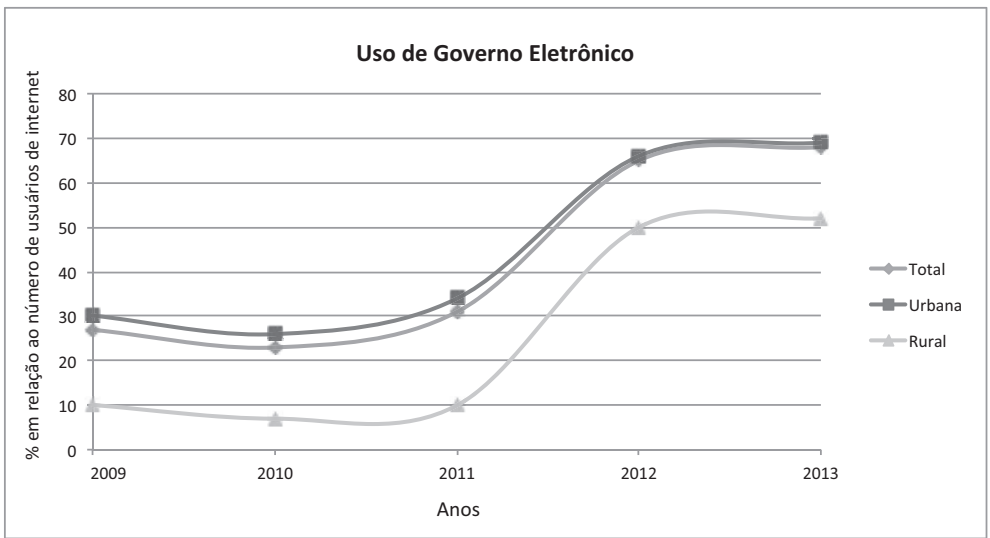

Fonte: elaborado pelos autores a partir de dados de CGI.br (2010, 2011, 2012, 2013, 2014). 
$\mathrm{Na}$ área urbana, 69\% dos indivíduos que acessam a internet em 2013 fizeram uso de serviços de governo eletrônico, enquanto que em 2009 esse percentual foi de $30 \%$, o que representa um aumento de $130 \%$. Na zona rural, houve também um aumento do uso, com uma diferença mais expressiva, passando de $10 \%$ em 2009 para $52 \%$ em 2013, atingindo um acréscimo de $420 \%$ no período. Salienta-se, no entanto, que o crescimento maior deu-se no período de 2011-2012. A Tabela 3 apresenta a evolução do uso dos serviços eletrônicos no período de 2009-2013.

Embora tenha havido um aumento na proporção de uso de governo eletrônico em geral no período 2009-2013, no que se refere aos serviços utilizados, estes apresentaram reduções de uso, algumas significativas: consulta de $\mathrm{CPF}$, de $49 \%$ para $27 \%$; declaração de imposto de renda, de $33 \%$ para $20 \%$; busca de informações sobre Previdência Social e benefícios sociais, de $22 \%$ para $17 \%$; busca de informações sobre serviços na área de educação, de $41 \%$ para $25 \%$; inscrição em concursos públicos, de 39\% para 23\%; busca de informações sobre empregos, de $33 \%$ para $17 \%$. Na direção oposta, o pagamento de impostos, multas e taxas sofreu um amuento, passando de $15 \%$ para $21 \%$.

Tabela 3 - Serviços de governo eletrônico utilizados

\begin{tabular}{lccccc}
\hline & \multicolumn{7}{l}{ \% EM RELAÇÃO AOS USUÁRIOS } \\
DE INTERNET & & & \\
& 2009 & 2010 & 2011 & 2012 & 2013 \\
\hline $\begin{array}{l}\text { 1. Obtenção de documentos } \\
\text { Consulta de CPF }\end{array}$ & 49 & 41 & 44 & 24 & 27 \\
$\begin{array}{l}\text { Busca de informações sobre emissão de } \\
\text { documentos (RG, CPF, etc.) }\end{array}$ & 29 & 29 & 34 & 21 & 27 \\
$\begin{array}{l}\text { Emissão de documentos (certificados, } \\
\text { atestados, comprovantes, etc.) }\end{array}$ & 21 & 16 & 26 & 13 & 18 \\
$\begin{array}{l}\text { Obtenção de certidões negativas, licenças e } \\
\text { permissões }\end{array}$ & 15 & 14 & 20 & 13 & 15 \\
\hline
\end{tabular}




\section{Pagamentos de taxas e impostos}

Declaração de imposto de renda (inclusive isentos)

33

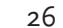

29

1920

Obtenção de informações sobre impostos

e taxas

27

22

26

16

Pagamento de impostos, multas e taxas

15

20

28

19

21

\section{Previdência e Assistência Social}

Busca de informações sobre Previdência

Social e benefícios sociais

24

19

24

15

17

Solicitação de serviços junto à Previdência Social

11

11

$14 \quad 08$

09

\section{Justiça e Segurança}

Consulta de pontos na carteira de habilitação /multas

21

19

$25 \quad 16$

Busca de informações sobre direito do consumidor

Consulta de andamento de atos processuais na justiça

$\begin{array}{lllll}13 & 12 & 18 & 11 & 12\end{array}$

Busca de informações sobre veículos roubados

$\begin{array}{lllll}09 & 05 & 07 & 05 & 05\end{array}$

Boletim de ocorrência

06

07

10

o6

06

\section{Outros Serviços}

Busca de informações sobre serviços públicos de educação

$\begin{array}{lllll}41 & 34 & 32 & 18 & 25 \\ 39 & 37 & 34 & 22 & 23 \\ 33 & 26 & 27 & 16 & 17\end{array}$

Busca de informações sobre empregos

Busca de informações sobre direitos do trabalhador

$\begin{array}{lllll}27 & 23 & 27 & 19 & 24\end{array}$

Busca de informações sobre serviços públicos de saúde

Matrículas em escolas ou instituições de ensino

$\begin{array}{lllll}14 & 12 & 14 & 08 & 10\end{array}$

Marcação e/ou agendamento de consultas médicas

Participação em fóruns, chats, votações etc. relacionados ao governo

$\begin{array}{lllll}09 & 07 & 10 & 06 & 08\end{array}$

Fonte: elaborado pelos autores a partir de dados de CGI.br (2010, 2011, 2012, 2013, 2014). 
Em relação à questão do não uso dos serviços eletrônicos, pesquisa TIC Domicílios 2013 revelou que 63\% dos não usuários de governo eletrônico apontam como motivo preferir fazer as consultas/contato pessoalmente. As características desses respondentes estão apresentadas no Gráfico 4. O não uso também está associado à dificuldade de encontrar os serviços ou até a própria complexidade inerente de alguns processos relativos aos serviços: $12 \%$ afirmam ser uma barreira a complicação para entrar em contato com a administração pública; $11 \%$ declararam que os serviços dos quais necessitavam não estavam disponíveis; e 14\% afirmam que os serviços desejados eram difíceis de serem encontrados. Um fato a destacar é que durante todo o período de 2009-2013, o item preferir fazer as consultas/contato pessoalmente mantem-se com nível alto de ocorrência (Tabela 4).

Gráfico 4 - Características dos não usuários de serviços de governo eletrônico

\begin{tabular}{|l|l|l|}
\hline \multicolumn{2}{|l|}{} & Prefiro fazer o contato pessoalmente \\
\hline \multirow{4}{*}{ TOTAL } & Sudeste & 63 \\
\hline \multirow{5}{*}{ Grau de instrução } & Nordeste & 67 \\
& Sul & 63 \\
& Norte & 59 \\
& Centro-Oeste & 49 \\
& Analfabeto / Educação infantil & 57 \\
\hline \multirow{5}{*}{ Faixa etária } & Fundamental & 71 \\
& Médio & 65 \\
& Superior & 62 \\
& De 16 a 24 anos & 60 \\
& De 25 a 34 anos & 63 \\
& De 35 a 44 anos & 67 \\
& De 45 a 59 anos & 68 \\
& 60 anos ou mais & 59 \\
\hline \multirow{5}{*}{ Classe social } & DE & 69 \\
& C & 63 \\
& B & 62 \\
& A & 56 \\
\hline \multirow{5}{*}{} &
\end{tabular}




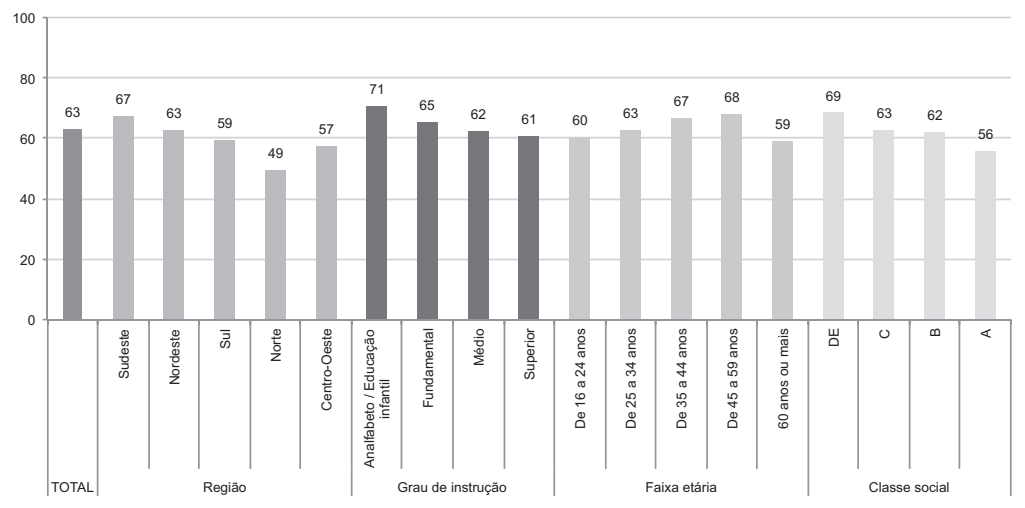

Fonte: CGI.br (2014).

Tabela 4 - Motivos para não uso de serviços de governo eletrônico

\begin{tabular}{|c|c|c|c|c|c|}
\hline \multirow{2}{*}{ MOTIVOS PARA NÃO UTILIZAÇÃO } & \multicolumn{5}{|c|}{$\begin{array}{l}\text { \% EM RELAÇÃO AOS USUÁRIOS DE } \\
\text { INTERNET }\end{array}$} \\
\hline & 2009 & 2010 & 2011 & 2012 & 2013 \\
\hline Prefere fazer o contato pessoalmente & 56 & 46 & 69 & 64 & 63 \\
\hline Serviços são difíceis de encontrar & 08 & 12 & 10 & 12 & 14 \\
\hline $\begin{array}{l}\text { Preocupação com a proteção e segurança } \\
\text { dos dados }\end{array}$ & 15 & 14 & 16 & 18 & 14 \\
\hline $\begin{array}{l}\text { Uso da internet para contato com a } \\
\text { administração pública é complicado }\end{array}$ & 12 & 09 & 11 & 13 & 12 \\
\hline Serviços desejados não estão disponíveis & 09 & 11 & 07 & 12 & 11 \\
\hline $\begin{array}{l}\text { Dificuldade em receber retorno às } \\
\text { solicitações }\end{array}$ & O3 & O3 & 04 & ०8 & ०9 \\
\hline $\begin{array}{l}\text { Serviços estão disponíveis. mas não é } \\
\text { possível completar a transação }\end{array}$ & $\mathrm{O} 2$ & $\mathrm{O} 2$ & 05 & 05 & 04 \\
\hline Não sabe / não respondeu & ०8 & 13 & 12 & 07 & $\mathrm{O} 2$ \\
\hline Outros motivos & 13 & 19 & - & - & - \\
\hline
\end{tabular}

Fonte: elaborado pelos autores a partir de dados de CGI.br (2010, 2011, 2012, 2013, 2014). 
Embora a disponibilidade de acesso via banda larga seja restrito ou inexistente em várias regiões, o custo do acesso com qualidade e velocidade à internet tem baixado, principalmente via rede de telefonia celular, o que tem contribuído para o aumento de usuários e, consequentemente, há um grande potencial para o uso dos serviços de governo eletrônico. No entanto, as questões segurança e privacidade ainda são consideradas fatores para não usá-los.

Ainda pode-se apontar como fatores restritivos ao uso a falta de conhecimento da disponibilidade dos serviços e da habilidade do uso por parte dos usuários, aspectos relacionados em parte com o nível de escolaridade e/ou condição socioeconômica e, consequentemente, dos processos de exclusão digital. Há, também, problemas decorrentes dos níveis de maturidade dos serviços (grau de execução das transações), na sua maioria apenas informacionais, não permitindo transações completas como, por exemplo, apuração e pagamento de impostos ou taxas. Também alguns serviços não estão disponíveis ou são difíceis de serem encontrados e, em alguns casos, existe uma demora ou inexistência do atendimento às solicitações dos usuários, o que é apontado pela pesquisa como motivos para o não uso dos serviços.

Para Santos e Reinhrad (2012), um fator que torna crítica a implementação dos serviços eletrônicos no Barsil é que o sistema de divisão de poderes no país se apresenta em três níveis verticais (federal, estadual e municipal), e cada um deles com divisões funcionais (executiva, legislativa e judicial). Com isto, oferecer serviços, principalmente àqueles que necessitam da integração entre os órgãos envolvidos, torna-se um processo complexo.

\section{CONSIDERAÇÕES FINAIS}

Este estudo teve como objetivo avaliar de forma exploratória o uso e as motivações do não uso de serviços de governo eletrônico no Brasil. Os dados apresentados pela pesquisa TIC Domicílios evidenciam um crescimento do acesso e uso da internet, mas sem o correlato incremento do uso dos serviços de governo eletrônico. 
Adicionalmente, foi constatado que existem vários motivos pelos quais os serviços não são usados o que indica a necessidade do estabelecimento de ações específicas para a redução desses limitadores, tais como a baixa capilaridade da infraestrutura de comunicações fora dos grandes centros, o custo do acesso à internet de alta velocidade e, principalmente, a resistência dos usuários, que ainda preferem, em sua maioria, obter os serviços e/ou informações presencialmente.

Como sugestão de pesquisas futuras, indica-se uma análise mais aprofundada sobre a ausência de correlação entre o aumento do uso de internet e o de serviços eletrônicos, e também uma exploração sobre a influência das características socioeconômicas e demográficas no uso destes serviços.

\section{REFERÊNCIAS}

AVGEROU, C. et al. The role of information and communication technology in building trust in governance: toward effectiveness and results. Washington, D.C.: Inter-American Development Bank, 2005.

BAUM, C.; DI MAIO, A. Gartner's four phases of e-government model. Stanford: Gartner Group, 2000. (Research Note)

BENT, S.; KERNAGHAN, K.; MARSON, D. Innovations and good practices in single-window service. Ottawa: Canadian Centre for Management Development, 1999.

CIBORRA, C.; NAVARRA, D. Good governance and development aid: risks and challenges of e-government in Jordan. In: KORPELA, M.; MONTEALEGRE, R.; POULYMENAKOU, A. Organizational Information Systems in the Context of Globalization. Dordrecht: Kluwer, 2003.

CGI.br. Pesquisa sobre o uso das tecnologias da informação e da comunicação no Brasil 2009: TIC domicílios e TIC empresas. São Paulo: Comitê Gestor da Internet no Brasil, 2010.

CGI.br. Pesquisa sobre o uso das tecnologias da informação e da comunicação no Brasil 2010: TIC domicílios e TIC empresas. São Paulo: Comitê Gestor da Internet no Brasil, 2011. 
CGI.br. Pesquisa sobre o uso das tecnologias da informação e da comunicação no Brasil 2011: TIC domicílios e TIC empresas. São Paulo: Comitê Gestor da Internet no Brasil, 2012.

CGI.br. Pesquisa sobre o uso das tecnologias da informação e da comunicação no Brasil 2012: TIC domicílios e TIC empresas. São Paulo: Comitê Gestor da Internet no Brasil, 2013.

CGI.br. Pesquisa sobre o uso das tecnologias da informação e da comunicação no Brasil 2012: TIC domicílios e TIC empresas. São Paulo: Comitê Gestor da Internet no Brasil, 2014.

CRIADO, J.; RAMILO, M. e-Administración: ¿un Reto o una Nueva Moda para las Administraciones del Siglo XXI? Algunos Problemas y Perspectivas de Futuro en torno a Internet y las Tecnologías de la Información y la Comunicación en las Administraciones Públicas. Revista Vasca de Administración Pública, v. 61, n. 1, p. 11-43, 2001.

DELOITTE; TOUCHE. The citizen as customer. CMA Management, v. 74 , n. 10, p. 58, Dec./Jan., 2000/2001.

EUROPEAN PUBLIC ADMINISTRATION NETWORK. Key principles of an interoperability architecture. Ireland, 2004.

FLAK, L. S.; SEIN M. K.; SÆB $\varnothing, ~ Ø$. Towards a cumulative tradition in e-government research: Going beyond the Gs and Cs. In: WIMMER, M. A.; SCHOLL, H. J.; GRÖNLUND, Å. (Ed.). EGOV 2007, LNCS 4656. Berlin: Springer-Verlag, 2007. p. 13-22.

GRANDE, J.; ARAUJO, M.; SERNA, M. La necesidad de teoría(s) sobre gobierno electrónico: uma propuesta integradora. In: CONCURSO DE ENSAYOS Y MONOGRAFÍAS DEL CLAD SOBRE REFORMA DEL ESTADO Y MODERNIZACIÓN DE LA ADMINISTRACIÓN PÚBLICA, 16., 2002, Caracas. Anais eletrônicos... Caracas: Centro Latinoamericano de Administración para el Desarollo, 2002.

HAGEN, M; KUBICEK, H. One-stop-government in Europe: results of 11 national surveys. Bremen: University of Bremen, 2000.

HILLER, J. S.; BÉLANGER, F. Privacy strategies for electronic government. In: ABRAMSON, M. A.; MEANS, G. E. (Ed.). E-government 2001. Lanham, MD: Rowman \& Littlefield Publishers, 200I. p. I62-I98. 
HOFFMAN, D.; NOVAK, T. P.; PERALTA, M. Building Consumer Trust Online. Communications of the ACM, v. 42, n. 4, p. 80-85, 1999.

IBGE. Censo Demográfico 2000. Disponível em: <http://www.sidra.ibge. gov.br>.

IBGE. Censo Demográfico 2010. Disponível em: <http://www.sidra.ibge. gov.br/>.

IBGE. Pesquisa Nacional por Amostra de Domicílios (PNAD). Disponível em< http://www.sidra.ibge.gov.br/pnad/default.asp $\geq$.

INTERNATIONAL TELECOMMUNICATION UNION. Manual for Measuring ICT Acess and Use by Households and Individuals - 2014 edition. Disponível em: <http://www.itu.int/dms_pub/itu-d/opb/ind/D-INDITCMEAS-2014-PDF-E.pdf>. Acesso em: 20 dez 2014.

INTOSAI. Auditing E-government. Viena: Intosai, Standing Committee on IT Audit, Task Force for Auditing E-Government, 2003.

JANSSEN, M.; CRESSWELL, A. An enterprise application integration methodology for e-government. Journal of Enterprise Information Management, v. 18, n. 5, p. 537-547, 2005.

KAMAL, M.; WEERAKKODY, V.; IRANI, Z. Analyzing the role of stakeholders in the adoption of technology integration solutions in UK local government: An exploratory study. Government Information Quarterly, v. 28, n. 2, p. 200-210, 2011.

LAYNE, K.; LEE, J. Developing fully functional e-government: a four stage model. Government Information Quarterly, v. 18, n. 2, p. 12-136, 2001.

LEE, J.; KIM, H. J.; AHN, M. J. The willingness of e-Government service adoption by business users: The role of offline service quality and trust in technology. Government Information Quarterly, v. 28, n. 2, p. 222-230, Apr. 2011.

MOON, M. The evolution of e-government among municipalities: rhetoric or reality? Public Administration Review, v. 62, n. 4, p. 424-433, July/Aug. 2002.

NATIONAL AUDIT OFFICE. Better public services through e-government. Londres: The Stationery Office, 2002. 
OECD. E-government: analysis framework and methodology. Paris:

OCDE, 2001.

OKOT-UMA, R. Electronic governance: re-inventing good governance. Londres: Commonwealth Secretariat London, 2001.

OLIVEIRA, C. Governo na era da informação: o caso do portal Bahia.gov. Lauro de Freitas: UNEB, 2003.

SANTOS, E. M.; REINHARD, N. Disponibilização e uso de serviços de governo eletrônico no Brasil: a visão dos usuários. In: PINHO, J. A. G. de. (Org.). Estado, sociedade e interações digitais: expectativas democráticas. Salvador: EDUFBA, 2012. p. 119-136.

SIAU, K.; LONG, Y. Innovations through information technology. Hershey: Idea Group, 2004.

SWID, A. A.; ELMELEGY, A. R. Management of e-services adoption in the telecommunications sector in Bahrain. Journal of Global Business and Technology, v. 8, n. 2, Fall, 2012.

TRANMÜLLER, R.; WIMMER, M. Processes - collaboration - norms - knowledge: Signposts for administrative application development. In: INTERNATIONAL WORKSHOP ON DATABASE AND EXPERT SYSTEMS APPLICATIONS, 11., 2000. Greenwich, UK. Proceedings... Los Alamitos: IEEE Computer Society, 2000. p. 1141-1145.

UNITED NATIONS. Global survey of e-Government. Nova York: United Nations; Division for Public Economics and Public Administration: American Society for Public Administration, 2001.

WORLD BANK. A definition of e-Government. World Bank's E-government Website. 2003. Disponível em: <http://www1.worldbank. org/publicsector/egov/definition.htm>. Acesso em: 16 jul. 2014. 